\title{
Score Evaluation Within the Extended Square-Root Information Filter
}

\author{
Maria V. Kulikova ${ }^{1}$ and Innokenti V. Semoushin ${ }^{2}$ \\ 1 School of Computational and Applied Mathematics, \\ University of the Witwatersrand, Private Bag 3, Wits 2050, \\ Johannesburg, South Africa \\ mkulikova@cam.wits.ac.za \\ 2 Ulyanovsk State University, 42 Leo Tolstoy Str., 432970 Ulyanovsk, Russia \\ i.semushin@ulsu.ru \\ http://staff.ulsu.ru/semoushin/
}

\begin{abstract}
A newly developed algorithm for evaluating the Log Likelihood Gradient (score) of linear discrete-time dynamic systems is presented, based on the extended Square-Root Information Filter (eSRIF). The new result can be used for efficient calculations in gradient-search algorithms for maximum likelihood estimation of the unknown system parameters. The theoretical results are given with the examples showing the superior perfomance of this computational approach over the conventional one.
\end{abstract}

\section{Introduction}

Consider the discrete-time linear dynamic stochastic system

$$
\begin{aligned}
x_{t+1} & =F_{t} x_{t}+G_{t} w_{t}, \quad t=0,1, \ldots, N \\
z_{t} & =H_{t} x_{t}+v_{t}, \quad t=1,2, \ldots, N
\end{aligned}
$$

with the system state $x_{t} \in \mathbb{R}^{n}$, the state disturbance $w_{t} \in \mathbb{R}^{q}$, the observed vector $z_{t} \in \mathbb{R}^{m}$, and the measurement error $v_{t} \in \mathbb{R}^{m}$, such that the initial state $x_{0}$ and each $w_{t}, v_{t}$ of $\left\{w_{t}: t=0,1, \ldots\right\},\left\{v_{t}: t=1,2, \ldots\right\}$ are taken from mutually independent Gaussian distributions with the following expectations:

$$
\left.\mathbf{E}\left\{\left[\begin{array}{c}
x_{0} \\
w_{t} \\
v_{t}
\end{array}\right]\right\}=\left[\begin{array}{c}
\bar{x}_{0} \\
0 \\
0
\end{array}\right], \quad \mathbf{E}\left\{\left[\begin{array}{c}
\left(x_{0}-\bar{x}_{0}\right) \\
w_{t} \\
v_{t}
\end{array}\right]\left[\begin{array}{c}
\left(x_{0}-\bar{x}_{0}\right) \\
w_{t} \\
v_{t}
\end{array}\right]\right]^{T}\right\}=\left[\begin{array}{ccc}
P_{0} & 0 & 0 \\
0 & Q_{t} & 0 \\
0 & 0 & R_{t}
\end{array}\right]
$$

and $\mathbf{E}\left\{w_{t} w_{t^{\prime}}^{T}\right\}=0, \mathbf{E}\left\{v_{t} v_{t^{\prime}}^{T}\right\}=0$ if $t \neq t^{\prime}$. Assume the system is parameterized by a vector $\theta \in \mathbb{R}^{p}$ of unknown system parameters. This means that all the above characteristics, namely $F_{t}, G_{t}, H_{t}, \bar{x}_{0}, P_{0} \geq 0, Q_{t} \geq 0$ and $R_{t}>0$, can depend upon $\theta$ (the corresponding notations $F_{t}(\theta), G_{t}(\theta)$ and so on, are suppressed for the sake of simplicity). 
Models like (11), (2), together with the associated Kalman filter, have been intensively used in many application fields such as control, communications, and signal/image processing. To apply the Kalman filter/smoother in the case of parametric uncertainty, it is necessary to identify the model, i. e., to estimate the unknown system parameters (or directly the Kalman filter/smoother parameters) from the available measurements $Z_{1}^{t}=\left(z_{1}, z_{2}, \ldots, z_{t}\right), t=1,2, \ldots, N$.

A very general and well known approach to parameter estimation is the maximum likelihood (ML) principle. The ML method for estimating $\theta$ requires the maximization of the Log Likelihood Function (LLF) $L_{\theta}\left(Z_{1}^{N}\right)$, which is the logarithm of the joint probability density of $z_{1}, z_{2}, \ldots, z_{N}$, with respect to $\theta$. It incorporates various gradient-search optimization algorithms. In this context, it is very important to find a means for efficient computation of Log Likelihood Gradient (LLG) known as the "score".

The first solutions to the problem [1,2] obtained by the direct differentiating the Kalman filtering equations are time consuming as they require the computation of $p$ vector filter sensitivity equations and $p$ matrix Riccati-type sensitivity equations, both run recursively in the forward time direction. An alternative approach was developed by Yared [3] who assumed a steady-state Kalman filter and used an adjoint filter, resulting in one additional backward pass in time. It works faster than the forward "differentiated" filter although at the expence of increased storage requirements. Wilson and Kumar simplified Yared's results [4] by moving from derivatives of Kalman variables to those of the original system matrices. They reduced the problem to solving a time-invariant matrix equation, an algebraic Riccati equation, a time-invariant Kalman filter equation, and the backward adjopint filter equation.

Segal and Weinstein developed LLG formulas for both the discrete and continuous-time systems [5, 6] using Fisher's identity and employing the Kalman smoother. Their approach enables to compute not only the LLG, but (approximately) the log-likelihood Hessian and the Fisher information matrix of $\theta$ as well. The continuous-time formula in [5] uses the Itô integral, which cannot be instrumented. For continuous-time case, Leland developed the improved formulas for the LLG [7, 8, free of this limitation.

Despite this remarkable success it is useful to develop alternative algorithms. The most important reason is that the previously mentioned methods suffer from some limitations mainly stemming from the use of the conventional Kalman filter (KF) implementation. This implementation - in terms of covariance matrices is particularly sensitive to roundoff errors [12]. As a consequence, any method for evaluating the log-likelihood gradient based on the conventional KF implementation, inherits this drawback and so cannot be considered as numerically stable. The alternative solutions to evaluate the LLG can be found in alternative KF implementation methods developed for dealing with the problem of numerical instability in many papers, for example [9, 10, 11], and described in [12].

In this paper we derive a new algorithm for evaluating the LLG based upon the extended Square-Root Information Filter (eSRIF) recently proposed in [11. Unlike the Conventional Kalman filter (CKF), the eSRIF avoids numerical 
instabilities arising from roundoff errors and has also the added feature of being better suited to parallel and to very large scale integration (VLSI) implementations (see [1]). Thus, inheriting these advantages, we expect our method to outperform the conventional KF mechanization for accuracy. These expectations will be verified by two examples of ill-conditioned problems.

The paper is organized as follows. In Section 2 we present a new algorithm for evaluating the LLG based upon the eSRIF. The comparison of the developed algorithm and the conventional approach in terms of sensitivity to roundoff errors is given in Section 3 . Section 4 presents some numerical results and finally, Section 5 concludes the paper.

\section{Log Likelihood Gradient Evaluation}

The Log Likelihood Function (LLF) of system (1), (2) is given by

$$
L_{\theta}\left(Z_{1}^{N}\right)=-\frac{1}{2} \sum_{t=1}^{N}\left\{\frac{m}{2} \ln (2 \pi)+\ln \left(\operatorname{det}\left(R_{e, t}\right)\right)+e_{t}^{T} R_{e, t}^{-1} e_{t}\right\}
$$

where $Z_{1}^{N}=\left(z_{1}, z_{2}, \ldots z_{N}\right)$ is $N$-step measurement history, $e_{t} \stackrel{\text { def }}{=} z_{t}-H_{t} \hat{x}_{t}$ is the zero-mean innovation sequence whose covariance is determined as $R_{e, t} \stackrel{\text { def }}{=}$ $\mathbf{E}\left\{e_{t} e_{t}^{T}\right\}=H_{t} P_{t} H_{t}^{T}+R_{t}$. The matrix $P_{t}$ is the error covariance matrix of the time updated estimate $\hat{x}_{t}$ of the state vector generated by the Kalman filter.

Let $l_{\theta}\left(z_{t}\right)$ denote the negative LLF for the $t$-th measurement $z_{t}$ in system (1), (2), given measurement history $Z_{1}^{t-1} \stackrel{\text { def }}{=}\left\{z_{1}, z_{2}, \ldots, z_{t-1}\right\}$, then

$$
l_{\theta}\left(z_{t}\right)=\frac{1}{2}\left\{\frac{m}{2} \ln (2 \pi)+\ln \left(\operatorname{det}\left(R_{e, t}\right)\right)+e_{t}^{T} R_{e, t}^{-1} e_{t}\right\} .
$$

From (3) one can easily obtain the expression for the LLG. Let $R_{e, t}=R_{e, t}^{T / 2} R_{e, t}^{1 / 2}$ where $R_{e, t}^{1 / 2}$ is a square-root factor of the matrix $R_{e, t}$ and $\bar{e}_{t}$ are the normalized innovations, i. e. $\bar{e}_{t}=R_{e, t}^{-T / 2} e_{t}$. Taking into account that the matrix $R_{e, t}^{1 / 2}$ is triangular, we can write down the expression

$$
\frac{\partial}{\partial \theta_{i}}\left[\ln \left(\operatorname{det}\left(R_{e, t}^{1 / 2}\right)\right)\right]=\operatorname{tr}\left[R_{e, t}^{-1 / 2} \frac{\partial\left(R_{e, t}^{1 / 2}\right)}{\partial \theta_{i}}\right] .
$$

Hence,

$$
\frac{\partial l\left(z_{t}\right)}{\partial \theta_{i}}=\operatorname{tr}\left[R_{e, t}^{-1 / 2} \frac{\partial\left(R_{e, t}^{1 / 2}\right)}{\partial \theta_{i}}\right]+\bar{e}_{t}^{T} \frac{\partial \bar{e}_{t}}{\partial \theta_{i}}, \quad i=1,2, \ldots, p
$$

as it follows directly from (3) and (4). 
According to the goal pursued by this research and stated in Section 1, we consider the eSRIF presented in [1]. For convenience, we reformulate it in the following form: given $P_{0}^{-T / 2}$ and $P_{0}^{-T / 2} \hat{x}_{0}=P_{0}^{-T / 2} \bar{x}_{0}$; calculate

$$
\begin{array}{r}
O_{t}\left[\begin{array}{ccc|c}
R_{t}^{-T / 2}-R_{t}^{-T / 2} H_{t} F_{t}^{-1} & R_{t}^{-T / 2} H_{t} F_{t}^{-1} G_{t} Q_{t}^{T / 2} & -R_{t}^{-T / 2} z_{t} \\
0 & P_{t}^{-T / 2} F_{t}^{-1} & -P_{t}^{-T / 2} F_{t}^{-1} G_{t} Q_{t}^{T / 2} & P_{t}^{-T / 2} \hat{x}_{t} \\
0 & 0 & I_{q} & 0
\end{array}\right] \\
=\left[\begin{array}{ccc|c}
R_{e, t}^{-T / 2} & 0 & 0 & -\bar{e}_{t} \\
-P_{t+1}^{-T / 2} K_{p, t} & P_{t+1}^{-T / 2} & 0 & P_{t+1}^{-T / 2} \hat{x}_{t+1} \\
* & * & *
\end{array}\right]
\end{array}
$$

where $O_{t}$ is any orthogonal transformation such that the matrix on the righthand side of formula (6) is block lower triangular. The matrix $P_{t}^{1 / 2}$ is a squareroot factor of $P_{t}$, i. e. $P_{t}=P_{t}^{T / 2} P_{t}^{1 / 2}, P_{t}^{1 / 2}$ is upper triangular. Similarly, we define $P_{t+1}=P_{t+1}^{T / 2} P_{t+1}^{1 / 2}, R_{t}=R_{t}^{T / 2} R_{t}^{1 / 2}, Q_{t}=Q_{t}^{T / 2} Q_{t}^{1 / 2}$ and $R_{e, t}=R_{e, t}^{T / 2} R_{e, t}^{1 / 2}$. For convenience we shall also write $A^{T / 2}=\left(A^{1 / 2}\right)^{T}, A^{-1 / 2}=\left(A^{1 / 2}\right)^{-1}$ and $A^{-T / 2}=\left(A^{-1 / 2}\right)^{T}$. Additionally, $K_{p, t}=F_{t} P_{t} H_{t}^{T} R_{e, t}^{-1}$.

It is easy to see that the LLG (5) in terms of eSRIF (6) is given by

$$
\frac{\partial l\left(z_{t}\right)}{\partial \theta_{i}}=-\operatorname{tr}\left[R_{e, t}^{1 / 2} \frac{\partial\left(R_{e, t}^{-1 / 2}\right)}{\partial \theta_{i}}\right]+\bar{e}_{t}^{T} \frac{\partial \bar{e}_{t}}{\partial \theta_{i}}, \quad i=1,2, \ldots, p
$$

To establish our algorithm for efficient evaluation of LLG (7) we prove the following result.

\section{Lemma 1. Let}

$$
Q A=L
$$

where $Q$ is any orthogonal transformation such that the matrix on the righthand side of formula (8) is lower triangular and $A$ is a nonsingular matrix. If the elements of $A$ are differentiable functions of a parameter $\theta$ then the upper triangular matrix $U$ in

$$
Q_{\theta}^{\prime} Q^{T}=U^{T}-U
$$

is, in fact, the upper triangular part of the matrix $Q A_{\theta}^{\prime} L^{-1}$.

Having applied Lemma 1 to eSRIF ([6), we obtain the following algorithm for computing LLG (7). 


\section{Algorithm LLG-eSRIF}

I. For each $\theta_{i}, i=1,2, \ldots, p$, calculate

$$
O_{t}\left[\begin{array}{ccc|c}
\frac{\partial}{\partial \theta_{i}}\left(R_{t}^{-T / 2}\right) & \frac{\partial}{\partial \theta_{i}}\left(S_{t}^{(1)}\right) & \frac{\partial}{\partial \theta_{i}}\left(S_{t}^{(2)}\right) & \frac{\partial}{\partial \theta_{i}}\left(S_{t}^{(3)}\right) \\
0 & \frac{\partial}{\partial \theta_{i}}\left(S_{t}^{(4)}\right) & \frac{\partial}{\partial \theta_{i}}\left(S_{t}^{(5)}\right) & \frac{\partial}{\partial \theta_{i}}\left(S_{t}^{(6)}\right) \\
0 & 0 & 0 & 0
\end{array}\right]=\left[\begin{array}{ccc|c}
X_{i} Y_{i} & M_{i} & L_{i} \\
N_{i} & V_{i} & W_{i} & K_{i} \\
* & * & * & *
\end{array}\right]
$$

where $O_{t}$ is the same orthogonal transformation as in (6) and

$$
\begin{gathered}
S_{t}^{(1)}=-R_{t}^{-T / 2} H_{t} F_{t}^{-1}, S_{t}^{(2)}=R_{t}^{-T / 2} H_{t} F_{t}^{-1} G_{t} Q_{t}^{T / 2}, S_{t}^{(3)}=-R_{t}^{-T / 2} z_{t}, \\
S_{t}^{(4)}=P_{t}^{-T / 2} F_{t}^{-1}, \quad S_{t}^{(5)}=-P_{t}^{-T / 2} F_{t}^{-1} G_{t} Q_{t}^{T / 2}, \quad S_{t}^{(6)}=P_{t}^{-T / 2} \hat{x}_{t} .
\end{gathered}
$$

II. For each $\theta_{i}, i=1,2, \ldots, p$, compute the matrix

$$
J_{i}=\left[\begin{array}{ccc}
X_{i} & Y_{i} & M_{i} \\
N_{i} & V_{i} & W_{i}
\end{array}\right]\left[\begin{array}{ccc}
R_{e, t}^{-T / 2} & 0 & 0 \\
-P_{t+1}^{-T / 2} K_{p, t} & P_{t+1}^{-T / 2} & 0 \\
* & * & *
\end{array}\right]^{-1} .
$$

III. For each $\theta_{i}, i=1,2, \ldots, p$, we split the matrices $J_{i}$ obtained at Step II as follows:

$$
J_{i}=\overbrace{\underbrace{\left[L_{i}+D_{i}+U_{i} \mid\right.}_{m+n} * * *]}^{m+n+q} \quad\}_{m+n}
$$

where $L_{i}, D_{i}$ and $U_{i}$ are the strictly lower triangular, diagonal and strictly upper triangular parts of matrix $J_{i}$, respectively.

IV. For each $\theta_{i}, i=1,2, \ldots, p$, compute the following quantities:

$$
\begin{gathered}
{\left[\begin{array}{cc}
\frac{\partial R_{e, t}^{-T / 2}}{\partial \theta_{i}} & 0 \\
-\frac{\partial\left(\tilde{P}_{t+1}^{-T / 2} K_{p, t}\right)}{\partial \theta_{i}} & \frac{\partial \tilde{P}_{t+1}^{-T / 2}}{\partial \theta_{i}}
\end{array}\right]=\left[L_{i}+D_{i}+U_{i}^{T}\right]\left[\begin{array}{cc}
R_{e, t}^{-T / 2} & 0 \\
-\tilde{P}_{t+1}^{-T / 2} K_{p, t} & \tilde{P}_{t+1}^{-T / 2}
\end{array}\right]} \\
\frac{\partial \bar{e}_{t}}{\partial \theta_{i}}=\left[\frac{\partial R_{e, t}^{-T / 2}}{\partial \theta_{i}}-X_{i}\right] R_{e, t}^{T / 2} \bar{e}_{t}+Y_{i} F_{t} \hat{x}_{t}-L_{i}, \\
\frac{\partial S_{t+1}^{(6)}}{\partial \theta_{i}}=\left[\frac{\partial\left(\tilde{P}_{t+1}^{-T / 2} K_{p, t}\right)}{\partial \theta_{i}}+N_{i}\right] R_{e, t}^{T / 2} \bar{e}_{t}+\left[\frac{\partial \tilde{P}_{t+1}^{-T / 2}}{\partial \theta_{i}}-V_{i}\right] F_{t} \hat{x}_{t}+K_{i} .
\end{gathered}
$$


Table 1. Comparison of Rounded Solutions to Problem 1 evaluated at the point $\theta=1$

\begin{tabular}{|c|c|c|}
\hline \multirow{2}{*}{$\begin{array}{c}\text { Filter } \\
\text { Implementation } \\
\end{array}$} & \multicolumn{2}{|c|}{ Solution } \\
\hline & Exact Answer & Rounded Answer \\
\hline $\begin{array}{l}\text { "differentiated" } \\
\text { Conventional } \\
\text { Covariance Filter }\end{array}$ & $\left.\left(P_{1}\right)_{\theta}^{\prime}\right|_{\theta=1}=\left[\begin{array}{cc}\frac{e^{2}}{1+e^{2}} & 0 \\
0 & 1\end{array}\right]$ & $\left.\left(P_{1}\right)_{\theta}^{\prime}\right|_{\theta=1} \stackrel{r}{=}\left[\begin{array}{ll}0 & 0 \\
0 & 1\end{array}\right]$ \\
\hline $\begin{array}{l}\text { "differentiated" } \\
\text { Conventional } \\
\text { Information Filter } \\
\end{array}$ & $\left.\left(P_{1}^{-1}\right)_{\theta}^{\prime}\right|_{\theta=1}=-\left[\begin{array}{cc}\frac{1+e^{2}}{e^{2}} & 0 \\
0 & 1\end{array}\right]$ & $\left.\left(P_{1}^{-1}\right)_{\theta}^{\prime}\right|_{\theta=1} \stackrel{r}{=}-\left[\begin{array}{cc}\frac{1}{e^{2}} & 0 \\
0 & 1\end{array}\right]$ \\
\hline $\begin{array}{l}\text { Algorithm LLG- } \\
\text { eSRIF }\end{array}$ & $\left.\left(P_{1}^{-1 / 2}\right)_{\theta}^{\prime}\right|_{\theta=1}=-\frac{1}{2}\left[\begin{array}{cc}\frac{\sqrt{1+e^{2}}}{e} & 0 \\
0 & 1\end{array}\right.$ & $\left.\left(P_{1}^{-1 / 2}\right)_{\theta}^{\prime}\right|_{\theta=1} \stackrel{r}{=}-\frac{1}{2}\left[\begin{array}{ll}\frac{1}{e} & 0 \\
0 & 1\end{array}\right]$ \\
\hline
\end{tabular}

V. Finally, compute the LLG according to (7).

Remark 1. Since, the matrices in (7) are triangular, only the diagonal elements of $R_{e, t}^{1 / 2}$ and $\frac{\partial\left(R_{e, t}^{-1 / 2}\right)}{\partial \theta_{i}}$ need to be computed. Hence, the Algorithm LLG-eSRIF allows the $m \times m$-matrix inversion of $R_{e, t}$ to be avoided in the evaluation of LLG.

\section{Ill-Conditioned Example Problems and Comparison}

To illustrate and compare the performance of the presented Algorithm LLGeSRIF and the conventional approach, i.e. a straightforward differentiation of the KF ("differentiated" KF), two simple test problems have been constructed.

Problem 1. Given:

$$
P_{0}=\left[\begin{array}{ll}
\theta & 0 \\
0 & \theta
\end{array}\right], H=[1,0], R=e^{2} \theta \quad \text { and } \quad F=I_{2}, Q=0, G=[0,0]^{T}
$$

where $\theta$ is an unknown parameter, $I_{2}$ is an identity $2 \times 2$ matrix, $0<e<<1$; to simulate roundoff we assume $e+1 \neq 1$ but $e^{2}+1 \stackrel{r}{=} 1$.

Calculate: $\left(P_{1}\right)_{\theta}^{\prime}$ at the point $\theta=1$.

For the $\theta=1$, this example illustrates the initialization problems that result when $H_{t} P_{t} H_{t}^{T}+R_{t}$ is rounded to $H_{t} P_{t} H_{t}^{T}$ (see, for example, 9]). The exact answer and the rounded answer, using $e^{2}+1 \stackrel{r}{=} 1$ in all calculations, are given for the "differentiated" CKF, "differentiated" Conventional Information filter (CIF) and the new Algorithm LLG-eSRIF developed in this paper (see Table 1). 
Table 2. Comparison of Rounded Solutions to Problem 2 evaluated at the point $\theta=1$

\begin{tabular}{l|c|c}
\hline \multicolumn{1}{c|}{$\begin{array}{c}\text { Filter } \\
\text { Implement }\end{array}$} & Solution \\
\cline { 2 - 3 } \begin{tabular}{l|c} 
"diff." \\
CKF
\end{tabular} & $\left.\left(P_{1}\right)_{\theta}^{\prime}\right|_{\theta=1}=\frac{1}{2+e^{2}}\left[\begin{array}{cc}1+e^{2} & -1 \\
-1 & 1+e^{2}\end{array}\right]$ & $\left.\left(P_{1}\right)_{\theta}^{\prime}\right|_{\theta=1} \stackrel{r}{=} \frac{1}{2}\left[\begin{array}{rr}1 & -1 \\
-1 & 1\end{array}\right]$ \\
\hline $\begin{array}{l}\text { "diff." } \\
\text { CIF }\end{array}$ & $\left.\left(P_{1}^{-1}\right)_{\theta}^{\prime}\right|_{\theta=1}=-\frac{1}{e^{2}}\left[\begin{array}{cc}1+e^{2} & 1 \\
1 & 1+e^{2}\end{array}\right]$ & $\left.\left(P_{1}^{-1}\right)_{\theta}^{\prime}\right|_{\theta=1} \stackrel{\underline{r}}{=}-\frac{1}{e^{2}}\left[\begin{array}{ll}1 & 1 \\
1 & 1\end{array}\right]$ \\
\hline $\begin{array}{l}\text { Algorithm } \\
\text { LLG- } \\
\text { eSRIF }\end{array}$ & $\left.\left(P_{1}^{-1 / 2}\right)_{\theta}^{\prime}\right|_{\theta=1}=-\frac{1}{2}\left[\begin{array}{cc}\sqrt{\frac{2+e^{2}}{1+e^{2}}} \frac{1}{e \sqrt{1+e^{2}}} \\
0 & \frac{\sqrt{1+e^{2}}}{e}\end{array}\right]$ & $\stackrel{r}{=}-\frac{1}{2}\left[\begin{array}{cc}\sqrt{2} & \frac{1}{e} \\
0 & \frac{1}{e}\end{array}\right]$ \\
\hline
\end{tabular}

It can be seen that all algorithms except the "differentiated" CKF give the nonsingular result. A singular answer will lead to a zero gain if a second measurement of the same type is processed.

Problem 2. Given: same as Problem 1, but $H=[1,1]$. Find: $\left(P_{1}\right)_{\theta}^{\prime}$ at the point $\theta=1$.

The exact solution and the rounded one for Problem 2 are summarized in Table2. With this more general type of ill-conditioning, only Algorithm LLG-eSRIF gives a nonsingular result.

\section{Numerical Results}

To substantiate the above theoretical result experimentally, we consider the example taken from [10]. Our simulation experiments are broken down into the following steps. Step 1 is planned to compute the negative LLF; in so doing, we compare our simulation results with those produced by the Conventional Information Filter (CIF). Step 2 is intended to compute the LLG; in this case, we compare the result generated by the Algorithm LLG-eSRIF with those produced by the "differentiated" CIF.

Example 1. Let the test system (11), (2) be defined as follows:

$$
\begin{gathered}
x_{t+1}=\left[\begin{array}{cc}
1 & \Delta t \\
0 & e^{-\Delta t / \tau}
\end{array}\right] x_{t}+\left[\begin{array}{l}
0 \\
1
\end{array}\right] w_{t}, \\
z_{t}=\left[\begin{array}{ll}
1 & 0
\end{array}\right] x_{t}+v_{t}
\end{gathered}
$$

where $\tau$ is a parameter which needs to be estimated. 

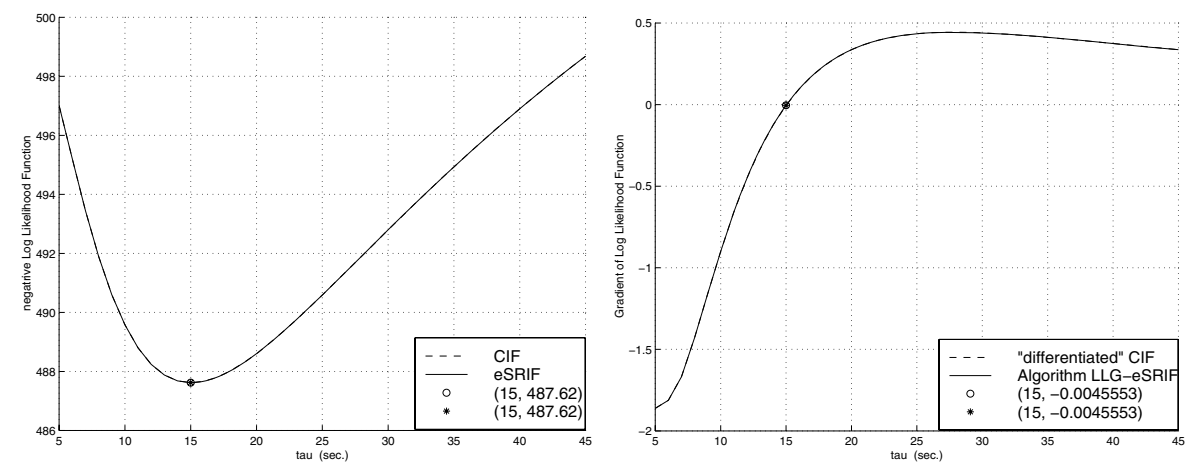

Fig. 1. The negative LLF and LLG calculated by the Conventional Information Filter, the extended Square-Root Information Filter, the "differentiated" Conventional Information Filter and Algorithm LLG-eSRIF, respectively.

The results of Step 1 and Step 2 are shown in Fig. 1. For the test problem, $\tau^{*}=15$ was chosen as the true value of parameter $\tau$. As can be seen, all two algorithms for evaluatinf the LLG produce exactly the same result and give the same zero point. Besides, it is readily seen that the estimate $\hat{\tau}_{\text {min }}$ minimizing the negative LLF coincides with the estimate $\hat{\tau}_{\text {grad }}$ at which the LLG is zero. Moreover, all estimates fit the true value of parameter $\tau$, i.e. $\tau^{*}=15$.

\section{Conclusion}

In this paper, the new algorithm for evaluating the Log Likelihood Gradient (score) of linear discrete-time dynamic systems has been developed. The necessary theory has been given and substantiated by the computational experiments. Two ill-conditioned example problems have been constructed to show the superior perfomance of the Algorithm LLG-eSRIF over the conventional approach.

\section{References}

1. G. C. Goodwin and R. L. Payne, Dynamic System Identification: Experiment Design and Data Analysis. New York: Academic, 1977.

2. R. L. Goodrich and P. E. Caines, Linear System Identification from Non-stationary Cross-sectional Data, IEEE Trans. Automat. Contr., vol. AC-24, pp. 403-411, June 1979.

3. K. I. Yared, On Maximum Likelihood Identification of Linear State Space models, Mass. Inst. Technol., Cambridge, MA, Rep. LIDS-TH-920, 1979.

4. D. A. Wilson and A. Kumar, Derivative Computations for the Log Likelihood Function, IEEE Trans. Automat. Contr., vol. AC-27, pp. 230-232, Feb. 1982.

5. M. Segal and E. Weinstein, A New Method for Evaluating the Log-Likelihood Gradient (Score) of Linear Dynamic Systems, IEEE Trans. Automat. Contr., vol. AC-33, pp. 763-766, Aug. 1988. 
6. M. Segal and E. Weinstein, A New Method for Evaluating the Log-Likelihood Gradient, the Hessian, and the Fischer Information Matrix for Linear Dynamic Systems, IEEE Trans. Automat. Contr., vol. AC-35, pp. 682-687, May 1989.

7. R. P. Leland, A New Formula for the Log-Likelihood Gradient for Continuous-Time Stochastic Systems, IEEE Trans. Automat. Contr., vol. AC-40, pp. 1295-1300, July 1995.

8. R. P. Leland, An Improved Log-Likelihood Gradient for Continuous-Time Stochastic Systems with Deterministic Input, IEEE Trans. Automat. Contr., vol. AC-41, pp. 1207-1210, Aug. 1996.

9. P.G. Kaminski, A.E. Bryson, S.F. Schmidt, Discrete Square Root Filtering: A survey of Current Techniques. IEEE Trans. on Aut. Cont. V. AC-16.(6), P. 727 $735,1971$.

10. G.J. Bierman, M.R. Belzer, J.S. Vandergraft, D.W. Porter, Maximum likelihood estimation using square root information filters. IEEE Trans. on Autom. Contr. 35(12), P. 1293-1298, 1990.

11. P. Park, T. Kailath, New square-root algorithms for Kalman filtering. IEEE Trans. on Autom. Contr. 40(5), P. 895-899, 1995.

12. M.S. Grewal, A.P. Andrews, Kalman Filtering: Theory and Practice. Prentice-Hall, Englewood Cliffs, New Jersey, 2001. 